\title{
The Impact of Aquatic Exercise on Sleep Behaviors in Children with Autism Spectrum Disorder
}

\author{
Jennifer Wood Kanupka ${ }^{1}$, Kathryn N. Oriel ${ }^{2, *}$, Cheryl L. George ${ }^{3}$, Lindi Crist ${ }^{1}$, \\ Kaitlyn Deardorff ${ }^{4}$, Danielle Douglass ${ }^{4}$, Kiersten Prenger ${ }^{4}$, Rebecca Ulicny ${ }^{4}$ \\ and Danielle Wirick ${ }^{4}$
}

${ }^{1}$ Lebanon Valley College, Department of Teacher Education, 101 N. College Ave. Annville, PA 17003, USA

${ }^{2}$ Lebanon Valley College, Department of Physical Therapy, 101 N. College Ave. Annville, PA 17003, USA

${ }^{3}$ St. Joseph's University, Department of Special Education, 5600 City Ave. Philadelphia, PA 19131, USA

${ }^{4}$ Students in the Doctor of Physical Therapy Program at Lebanon Valley College, USA at the Time of the Study

\begin{abstract}
Children with Autism Spectrum Disorder (ASD) often demonstrate disturbed sleep behaviors that negatively impact daytime behavior. The purpose of this study was: 1) To determine if participation in aquatic exercise improves sleep duration in children with ASD, and 2) to examine the social validity of the intervention. A within-subjects crossover design over an 8 week period was used. Participants were assigned to the intervention (aquatic exercise $2 x /$ week for 1 hour) or the control (no exercise) condition for 4 weeks; they then switched conditions for 4 weeks. Sleep habits were recorded using the EMFIT QS sleep monitor. The IRP-15 was administered to parents after completion of the intervention to establish social validity. Mean sleep duration for intervention versus control was compared using the Wilcoxon-Signed Ranks Test. Statistically significant improvements $(p=0.012)$ in sleep duration were observed (intervention: 9.27 hours vs control 8.37 hours). Descriptive statistics were used to analyze IRP-15 data indicating the majority of parents strongly agreed that aquatic exercise had a positive effect on their child's sleep.
\end{abstract}

Results suggest that participation in an aquatic exercise program may lead to improved sleep duration in children with $A S D$, and is a socially valid intervention.

Keywords: Autism Spectrum Disorder, sleep, aquatic exercise.

\section{INTRODUCTION}

Sleep disturbances are the most burdensome complaint among parents of children with Autism Spectrum Disorder (ASD). According to Knight and Johnson [1], 66-86\% of children with ASD experience sleep disturbances. The most commonly reported sleep problems include prolonged sleep latency, decreased sleep efficiency, frequent wakings, wakings of longer duration, reduced total sleep time, bedtime resistance, and daytime sleepiness [1,2]. Sleep disturbances often persist as children move into adolescence, although the types of problems they experience changes slightly. Younger children tend to experience more bedtime resistance, sleep anxiety, and night wakings, whereas adolescents and older children tend to have more difficulty falling asleep, staying asleep, and daytime sleepiness [3].

A significant association exists between sleep problems and the severity of ASD symptoms. Poor sleep patterns are especially problematic for children and adolescents with ASD because it can exacerbate

*Address correspondence to this author at $101 \mathrm{~N}$. College Ave., Annville, PA 17003, USA; E-mail: oriel@Ivc.edu problematic daytime behaviors, including repetitive and stereotypic behaviors, hyperactivity, inattention, social skill deficits, communication impairments, aggression, irritability, tantrums, self-injury, and affective problems [2]. All of these behaviors negatively impact learning, cognition, family functioning, and other aspects of daily life [4]. Additionally, Hodge, Hoffman, Sweeney, and Riggs [5] found that the sleep of children with ASD significantly predicted maternal mental health issues, decreased maternal sleep, and increased maternal stress levels.

Various methods to decrease sleep disturbances in children with ASD have been described in the literature. Cohen et al. [2] described parent-based educational and behavioral interventions. This included sleep hygiene approaches which focused on setting a bedtime, creating a bedtime routine, and reducing emotional and behavioral stimulation at night such as watching television. Knight and Johnson [1] described a "behavioral treatment package" including: circadian rhythm management with set sleep and wake times where naps are avoided; positive bedtime routines including 4-6 quiet, calm activities before bedtime; use of a white noise machine on 50-75 dB during sleep; and graduated extinction where parents wait 
progressively longer before checking on the child during tantrums, then leaving while the child is still awake. This study found a decrease in night wakings and sleep latency, as well as parent satisfaction with the treatment program. Melatonin has also been used as a pharmacological method to reduce sleep disturbances. Malow et al. [6] found that melatonin improved sleep latency in prepubertal children with ASD, but did not see improvement in sleep duration or night wakings. Wright et al. [7] had similar findings of improved sleep latency as well as total sleep time, but saw no effect on number of night wakings with the use of melatonin.

Brand et al. [8] examined the influence exercise had on sleep with 434 typically developing adolescents; 258 were student athletes and the control group consisted of 176 students who were not physically active. The student athletes participated in sports such as volleyball, skiing, track and field, soccer, swimming, ice hockey, martial arts, and fencing. The student athletes reported exercising 17.69 hours per week versus 4.69 hours for the adolescents in the control group. All participants completed sleep-related questionnaires and sleep logs for 7 consecutive days. The results of the study found that the athletes engaged in routine exercise experienced increased sleep quality, in conjunction with decreased tiredness and improved concentration during the day. The researchers suggested that exercise leads to physiological changes that facilitate homeostatic sleep regulation, which then acutely stabilizes circadian rhythms and reduces drowsiness during the day. Similarly, a meta-analysis by Iftikhar, Kline and Youngstedt [9] analyzed data from several studies that found a positive relationship between self-reported exercise and self-reported sleep quality. There were significant improvements in sleep efficiency, daytime sleepiness, and a significant reduction in severity of obstructive sleep apnea (OSA) across all studies examined [9].

Physical activity has also been used in attempt to decrease sleep disturbances in children with ASD. Due to the prevalence of sleep disturbances in children with ASD, Brand et al. [8] researched the effects of physical activity, specifically aerobic exercise training, and motor skills training, on sleep patterns in children with ASD. Though the intervention was only 3 weeks long, there were noted benefits on sleep on the nights following physical activity compared to the nights without physical activity [8]. Specifically, these findings indicated that sleep parameters such as sleep efficiency, wake time after sleep onset, and shorter sleep onset latency were improved (8). Similarly, Oriel et al. [10] examined the impact of aquatic exercise on sleep in children with ASD. The results showed that participants fell asleep an average of 17.25 minutes faster and stayed asleep an average of 83.2 minutes longer following aquatic exercise compared to a control phase. These findings suggested that participation in an aquatic exercise program may lead to improved sleep habits in children with ASD.

In addition to providing benefits in sleep habits, aquatic exercise programs have also been shown to provide advantages in other aspects of health. A study by Chien-Yu Pan [11] was conducted in 16 boys with a diagnosis of ASD, ages ranging from 6 to 9 years old, and were able to follow instructions. This study documented improvements in decreased antisocial behavior and an improvement in aquatic skills [11]. A recent systematic review by Mortimer, Privopoulos, and Kumar [12] found improvements in children with ASD in both social interactions and behaviors as well as a decrease in stereotypical movement, improved emotional functioning, and increase in better school behavior following hydrotherapy. In addition, FragalaPinkham, Haley, and O'Neil [13] conducted a study in which children with ASD participated in group aquatic therapy. The patient population consisted of children ages 6-11, with the diagnosis of ASD. The findings of this study concluded that group aquatic therapy programs provide a fun, low impact option to improve cardiorespiratory endurance, and allow children to achieve the recommended time for physical activity [13].

While preliminary evidence suggests that aquatic exercise may improve sleep habits in children with ASD, additional research is necessary to further strengthen these hypotheses. The purpose of this study was: 1) to determine if children with ASD who participate in an aquatic exercise program have improvements in sleep duration, and 2) to examine the social validity of aquatic exercise as an intervention to improve sleep duration in children with ASD.

\section{METHODS}

\subsection{Participants}

The participants in this study were recruited through a local ASD support group and flyers sent to local ASD support teachers. In order to be included in the study, participants had to be formally diagnosed with ASD. The participants included eight children, ages 4 years 
Table 1:

\begin{tabular}{|c|c|c|c|c|c|}
\hline Participants & Age & Gender & $\begin{array}{l}\text { CSHQ } \\
\text { (out of 66) }\end{array}$ & Medication & $\begin{array}{l}\text { Adult: Participant Ratio and Swimming } \\
\text { Skills }\end{array}$ \\
\hline Participant 1 & 7 y 10 mo & M & 37 & None & $\begin{array}{l}\text { 1:1 - supervision; age appropriate swimming } \\
\text { skills }\end{array}$ \\
\hline Participant 2 & 4 y 10 mo & M & 50 & None & $\begin{array}{c}\text { 2:1 - moderate assist; required trunk flotation } \\
\text { device }\end{array}$ \\
\hline Participant 3 & 8 y 9 mo & M & 45 & $\begin{array}{l}\text { Risperdal, Temex, } \\
\text { Escitalopram }\end{array}$ & $\begin{array}{l}2: 1 \text { - age appropriate swimming skills; } \\
\text { increased assist for behavioral issues }\end{array}$ \\
\hline Participant 4 & 6 y 2 mo & M & 37 & Albuterol, daily vitamin & $\begin{array}{l}\text { 1:1 - minimal assist; required flotation } \\
\text { devices on (B) upper extremities }\end{array}$ \\
\hline Participant 6 & 6 y 6 mo & M & 44 & Cetirizine & $\begin{array}{l}\text { 1:1 - supervision; age appropriate swimming } \\
\text { skills }\end{array}$ \\
\hline Participant 7 & 10 y 3 mo & M & 33 & None & $\begin{array}{c}2: 1 \text { - moderate assist; required flotation } \\
\text { device; increased assist for behavioral issues }\end{array}$ \\
\hline
\end{tabular}

and 10 months to 13 years and 4 months (58 months to 160 months), with ASD. The average age of participants was 8.08 years, or 97.7 months. Table 1 describes the participant demographics.

\subsection{Design}

This study utilized a within-subjects crossover design throughout an 8 week period. Each participant received 4 consecutive weeks of the intervention (aquatic exercise 2x per week, 1 hour session), and 4 consecutive weeks as the control (no aquatic exercise). Seven participants received the intervention for the initial 4 weeks, while ten of participants received the intervention for the second 4 weeks. The crossover design decreases between participant variability and allows each participant to receive the intervention while acting as his own control (14). By recording sleep habits throughout the entire 8 weeks, the crossover design would demonstrate if the intervention caused the desired outcome, specifically improved sleep habits.

\subsection{Measures}

\section{Baseline Measures}

The Children's Sleep Habit Questionnaire (CSHQ) was completed by parents prior to participating in the intervention.

\section{CSHQ}

In this study, the Children's Sleep Habit Questionnaire (CSHQ) was used as a subjective measure of each participant's sleep habits. The Children's Sleep Habit Questionnaire (CSHQ) is a retrospective 45-item parent questionnaire developed by clinical researchers at Brown University that was used to examine the sleep behavior in participants at the beginning of the study [15]. This survey instrument consists of 8 subscales and 33 items. A total CSHQ score of 41 has been determined to be the clinical cutoff for identifying children with probable sleep disturbances [16]. This tool has demonstrated to be useful for screening purposes, tracking treatment effects, and assessing the presence of sleep disorders in children 4 to 10 years old with neurodevelopmental disorders. A study by Goodlin-Jones et. al [16] found that there was a significant, yet relatively small, correlation between CSHQ scores and parent sleep logs. This finding is to be expected due to the fact that the parents' knowledge of their child's sleep habits may differ from any objective measurements. It was also determined that 30 out of the 33 items were statistically significant $(p<.001)$, making this CSHQ instrument a valid measure to quantify sleep problems.

\section{Outcome Measures}

\section{Sleep Habits}

Various methods and tools, both subjective and objective, can be utilized to gather data on sleep habits, providing data to be compared and analyzed. In terms of accuracy and stability, other methods to measure sleep habits, such as actigraphy, are recommended over sleep diaries [17]. Interview procedures, questionnaires, and diaries are based on subjective information, whereas actigraphy records objective measures of the child's sleep-wake patterns derived from movements. Although actigraphy provides 
high sensitivity, the detection of wakefulness during sleep episodes is relatively poor with certain devices, some scoring algorithms, and specific populations. Due to the limitations of actigraphy, it is recommended to use complementary assessment methods including subjective and objective information whenever possible [17]. Sleep habits were recorded throughout the duration of the study with the use of sleep diaries and sleep monitors.

\section{Emfit Device}

A non-contact sleep monitor was utilized to collect quantitative data on the sleep behavior of the participants. The non-contact monitor (Emfit device) is an ultra-thin durable sensor that is placed under the mattress of the participant. The sensor is then plugged into a wall outlet and then connected to the home's wifi. Each participant's family was provided with a monitor to install in their home setting. Once installed, the sensor then sends data to a wifi enabled application in which quantitative data was stored such as heart rate, respiratory rate, movement, total sleep time, sleep latency, and number of awakenings via a cloud based server. In previous studies aimed to compare Emfit bed sensors to the gold standard of laboratory PSG, the mattress algorithm showed an agreement ranging from $71 \%-79 \%$ with PSG data in determining wake, NREM, and REM sleep states [18].

\section{Sleep Diaries}

Sleep diaries are used as a method to collect sleep information and sleep habits because it is convenient and economical, hence why sleep diaries were chosen as a supplemental measurement tool for sleep habits of the participants in our study in addition to the Emfit sleep monitor. An interview protocol was established via telephone calls to the parent/guardian biweekly, following the nights the participants participated in the aquatic program (twice weekly). The interview protocol consisted of the following questions: What time did your child go to bed last night? How many minutes did it take for him/her to fall asleep? How many times did your child wake up last night? On average how long was he/she awake each time? What time did your child wake up this morning? Is there anything else you would like to tell me about your child's sleep last night?

\section{Social Validity (IRP 15)}

\section{Intervention Rating Profile 15}

In order to measure the effectiveness and parent perception of the aquatic intervention, the Intervention Rating Profile 15 was used by this study. The
Intervention Rating Profile (IRP-15) is a shortened version of the Intervention Rating Profile that entails 15 statements on varying aspects of intervention acceptability [19]. The assessment uses a 6-point Likert-type scale where subjects rate their level of agreement or disagreement with each statement [19]. Obtaining higher scores with this instrument is associated with acceptable interventions. A moderate level of acceptability would require a total summed score of 52.5 [20]. Using Cronbach's alpha, the reliability of the IRP-15 demonstrated to be .98 [19]. The study by Martens et. al completed ANOVAS indicating statistically significant $(p<.001)$ main effects for behavior problem severity and intervention with the IRP-15 [19]. The IRP-15 was modified for use in this study. Specifically, parents were told in the instructions that the word "intervention" in the statements was referring to "aquatic exercise", and sleep disturbances was inserted where appropriate. For example, question 14 on the IRP-15 read, "This intervention was a good way to handle my child's sleep disturbances."

\subsection{Program Description}

Before the official start of study, the researchers met with interested parents/guardians of the participants to discuss the study parameters, and answer questions as needed. Every parent/guardian completed the previously mentioned outcome measures, was provided information related to the demographic information of the child and other necessary release forms. The parents/guardians were given an Emfit device and instructed on how to install and utilize the device.

The intervention phase involved a pediatric aquatic exercise program that was consistent with a previous established aquatic therapy program [10]. Most participants were paired with an adult in a 1:1 ratio, but some participants had 2:1 ratios based on more assistance needed or an increased number of adults to children ratio. One to two researchers remained outside the pool during the aquatic exercise to instruct the participants during the session.

Each aquatic session lasted 60 minutes and had a consistent structure. The session began with a warmup which consisted of walking in a circle with the group clockwise and counterclockwise. After the warm-up was complete, each participant performed specific upper and lower body exercises. Upper extremity exercises included forward/backward arm circles, punches, shoulder abduction/adduction, and bicep 
curls. Lower extremity exercises included wall kicks, marching in place, hip abduction, and push-offs of the wall. Next, the participants partook in various cardiovascular exercises, including jumping jacks, jogging in place, and kickboard relays. Upon completion of all cardiovascular exercises, the participants then participated in game, red light-green light, back and forth the length of the pool. The session concluded with free swim, a time where participants played with various pool toys, swimming noodles, and could swim as they pleased. Every participant was encouraged to remain as active as possible throughout the duration of the session, but more so during the free swim time. At the conclusion of free swim, a cool-down was completed, which was the same as the warm-up at the beginning of the session.

\subsection{Statistical Analysis}

Descriptive statistics were used to summarize participant demographic data along with the data from the IRP-15. The Wilcoxon Signed-Rank test was used to determine if mean differences existed between the intervention and control phases of the study. This nonparametric statistic was used due to the small sample size. A Pearson correlation was used to determine if agreement existed between the data of Emfit and sleep diaries.

\section{RESULTS}

\section{Participant Demographics}

The average age of our participants was 97.71 months (8.08 years). The mean CSHQ score for participants was 37.42 . None of the participants were on sleep medication throughout the study.

\section{Sleep}

\section{Emfit}

Wilcoxon Signed-Rank test was used to determine if significant differences existed for total sleep on intervention versus control days. A statistically significant improvement was observed on intervention days $(p=0.12)$. The mean total sleep time of participants was 555.955 minutes (9 hours 16 minutes) on intervention days versus a mean of 502.268 minutes ( 8 hours and 22 minutes) on control days. Overall, participants slept 53.69 minutes longer on intervention versus control days. A significant difference was not observed for the various stages of sleep (i.e. deep sleep versus light sleep).

\section{Sleep Diaries}

Wilcoxon Signed-Rank test was used to determine if significant differences existed for total sleep on intervention versus control days. A statistically significant difference was not observed $(p=0.484)$. The mean total sleep time of participants was 536.305 minutes (8 hours 56 minutes) on intervention days versus a mean of 550.25 minutes ( 9 hours and 10 minutes) on control days. Per parent report, participants slept 13.95 minutes longer on control days versus intervention days.

\section{Emfit versus Sleep Diaries}

Pearson correlations were used for total sleep time derived from the Emfit and sleep diaries. Statistically significant correlation coefficients were not observed when comparing the two methods $(p=0.834)$.

\section{Social Validity}

\section{Attendance}

The mean attendance for eight aquatic sessions was $84.4 \%(n=8)$. Three participants attended all eight sessions, two participants attended seven of eight sessions, one participant attended six of eight sessions, and two participants attended five of eight sessions.

\section{IRP-15}

The IRP-15 scores ranged from 58 to 90 (mean $=$ 80.4). All scores are above the 52.5 cut-off score, indicating that parents perceived aquatic exercise to be an effective intervention for children with ASD.

\section{DISCUSSION}

This study examined whether children with ASD would have improved sleep habits, specifically, total sleep duration, time to fall asleep, and number of awakening following the participation in an aquatic exercise program. The finding of improved total sleep time is consistent with the previous study by Oriel et al. [10]. Both studies found that participants slept longer following aquatic exercise. In comparison to Oriel et al. [10] where they found an increase of 83.2 minutes of time slept, the current study found only a modest increase time of sleep of 53.6 minutes. Although these studies showed an improvement after completion of aquatic exercise, different measurement tools were utilized to obtain these findings. Sleep diaries were used in both studies, however this study did not find a statistically significant difference in total sleep between 
the intervention and control. This is inconsistent with the findings in Oriel et al. [10] which found a statistically significant improvement in sleep time based on the sleep diaries.

In this study, no correlation was found between measuring sleep with sleep diaries and the Emfit devices. This finding is not consistent with previous studies comparing sleep diaries and actigraphy data for children with sleep disturbances. In a sample of infants with sleep disturbances, Sadeh [21] found high correlations for sleep onset and sleep duration between actigraphy and diary data. In another study, Asaka and Takada [17] found high correlations between diary and actigraphy data for sleep onset, offset, and duration, but much lower correlations for night wakes and wakes after sleep onset. Similarly, a longitudinal study on infant's sleep patterns in their first 12 months of life, So, Adamson, and Horne [22] reported no difference between actigraphs and sleep diaries for total percentage of sleep or wake time over twenty-four hours [23].

Similar to findings in this study, a study performed by Vallières and Morin [24] found that actigraphy and sleep diaries were not correlated as actigraphy underestimated sleep-onset latency while the sleep diary overestimated it as compared to polysomnography- the gold standard for measuring sleep. Actigraphy data was more accurate than sleepdiary data when compared to polysomnography. Finally, actigraphy was sensitive in detecting the effects of treatment on several sleep parameters and is recommended to be used in conjunction with sleep diaries.

The poor correlation between actigraphy and subjective reports for measuring sleep quality in our study could be attributed to either the inaccuracy of the Emfit device algorithm, inaccuracy of subjective reports as reported in the sleep diaries, or a combination of both.

Several limitations existed which limits the generalizability of the results. One challenge was the set-up of the Emfit sensors, as well as ensuring they were working throughout all phases of the study. The Emfit sensors required that parents set them up using a manual provided to them; many parents were unable to do so even with continued support and reminders from the researchers. Difficulty with the monitors led to the small sample (eight participants) in our study. Additionally, any potential long term impact of the aquatic exercise program on sleep habits was not measured.

To strengthen this body of research, future studies should include a larger sample size and also an extended intervention period. It may also be beneficial to allow time for a trial period for the Emfit sensor to ensure it is working properly and collecting data.

\section{CONCLUSION}

In conclusion, this study provides additional evidence supporting the participation in aquatic exercise for improving sleep behaviors in children with ASD. The participants in our study slept longer on nights following participation in aquatic exercise. Clinical relevance is demonstrated for aquatic exercise should be considered as a safe and effective strategy to improve sleep habits in children with ASD. Pediatric physical therapists should recommend this intervention when appropriate, and consider developing communitybased aquatic exercise programs in needed areas.

Further research is necessary to strengthen the body of evidence in support of aquatic exercise to improve sleep disturbances in children with ASD. However, aquatic exercise should be considered as a safe and effective intervention to improve sleep habits in children with ASD.

\section{REFERENCES}

[1] Knight R, Johnson C. Using a behavioral treatment package for sleep problems in children with autism spectrum disorders. Child \& Family Behavior Therapy 2014; 36(3): 204-221.

https://doi.org/10.1080/07317107.2014.934171

[2] Cohen S, Conduit R, Lockley S, Rajaratnam S, Cornish K. The relationship between sleep and behavior in autism spectrum disorder (ASD): A review. Journal of Neurodevelopmental Disorders 2014; 6(1): 44. https://doi.org/10.1186/1866-1955-6-44

[3] Goldman S, Richdale A, Clemons T, Malow B. Parental sleep concerns in autism spectrum disorders: Variations from childhood to adolescence. Journal of Autism \& Developmental Disorders 2012; 42(4): 531-538. https://doi.org/10.1007/s10803-011-1270-5

[4] Engelhardt C, Mazurek M, Sohl K. Media use and sleep among boys with autism spectrum disorder, ADHD, or typical development. Pediatrics 2013; 132(6): 1081-1089. https://doi.org/10.1542/peds.2013-2066

[5] Hodge D, Hoffman C, Sweeney D, Riggs M. Relationship between children's sleep and mental health in mothers of children with and without autism. Journal of Autism \& Developmental Disorders 2013; 43(4): 956-963. https://doi.org/10.1007/s10803-012-1639-0

[6] Malow B, Adkins K, McGrew S, Wang L, Goldman S, Fawkes $D$, Burnette C. Melatonin for sleep in children with autism: A controlled trial examining dose, tolerability, and outcomes. Journal of Autism and Developmental Disorders 2012; 42(8): 1729-1737.

https://doi.org/10.1007/s10803-011-1418-3 
[7] Wright B, Sims D, Smart S, Alwazeer A, Alderson-Day B, Allgar V., . . . Jardine J. Melatonin versus placebo in children with autism spectrum conditions and severe sleep problems not amenable to behaviour management strategies: $A$ randomised controlled crossover trial. Journal of Autism and Developmental Disorders 2011; 41(2): 175-184. https://doi.org/10.1007/s10803-010-1036-5

[8] Brand S, Gerber M, Beck J, Hatzinger M, Pühse U, HolsboerTrachsler E. High exercise levels are related to favorable sleep patterns and psychological functioning in adolescents: a comparison of athletes and controls. Journal of Adolescent Health 2010; 46(2): 133-141. https://doi.org/10.1016/j.jadohealth.2009.06.018

[9] Iftikhar I, Kline C, Youngstedt S. Effects of exercise training on sleep apnea: A meta-analysis. Lung 2014; 192(1): 17584. https://doi.org/10.1007/s00408-013-9511-3

[10] Oriel K, Kanupka J, DeLong K, Noel K. The impact of aquatic exercise on sleep behaviors in children with Autism Spectrum Disorder: A pilot study. Focus on Autism and Other Developmental Disabilities 2016; 31(4): 254-261. https://doi.org/10.1177/1088357614559212

[11] Pan C. Effects of water exercise swimming program on aquatic skills and social behaviors in children with autism spectrum disorders. Autism: The International Journal Of Research And Practice 2010; 14(1): 9-28. https://doi.org/10.1177/1362361309339496

[12] Mortimer R, Privopoulos M, Kumar S. The effectiveness of hydrotherapy in the treatment of social and behavioral aspects of children with autism spectrum disorders: A systematic review. Journal of Multidisciplinary Healthcare 2014; 793-104.

[13] Fragala-Pinkham M, Haley S, O'Neil M. Group aquatic aerobic exercise for children with disabilities. Developmental Medicine And Child Neurology 2008; 50(11): 822-827. https://doi.org/10.1111/j.1469-8749.2008.03086.x

[14] Jones B, Kenward G. Design and analysis of crossover trials. London, England: Chapman \& Hall 2003.
[15] Owens J, Spirito A, McGuinn M. The Children's Sleep Habits Questionnaire (CSHQ): Psychometric properties of a survey instrument for school-aged children. Sleep 2001; 23(8): 1043-51

[16] Goodlin-Jones B, Sitnick S, Tang K, Liu J, Anders T. The Children's Sleep Habits Questionnaire in toddlers and preschool children. Journal of Developmental \& Behavioral Pediatrics 2008; 29(2): 82-88. https://doi.org/10.1097/DBP.0b013e318163c39a

[17] Asaka Y, Takada S. Comparing sleep measures of infants derived from parental reports in sleep diaries and acceleration sensors. Acta Paediatrica 2011; 100(8): 11581163.

https://doi.org/10.1111/j.1651-2227.2011.02204.x

[18] Kelly J, Strecker R, Bianchi M. Recent developments in home sleep-monitoring devices. ISRN neurology 2012.

[19] Martens B, Witt J, Elliott S, Darveaux D. Teacher judgments concerning the acceptability of school-based interventions. Professional Psychology: Research and Practice 1985 16(2): 191. https://doi.org/10.1037/0735-7028.16.2.191

[20] Carter S. The social validity manual: A guide to subjective evaluation of behavior interventions. Academic Press 2009.

[21] Sadeh A. The role and validity of actigraphy in sleep medicine: an update. Sleep Medicine Reviews 2011; 15(4): 259-267. https://doi.org/10.1016/j.smrv.2010.10.001

[22] So K, Adamson T, Horne R. The use of actigraphy for assessment of the development of sleep/wake patterns in infants during the first 12 months of life. Journal of Sleep Research 2007; 16(2): 181-187. https://doi.org/10.1111/j.1365-2869.2007.00582.x

[23] Hall W, Liva S, Moynihan M, Saunders R. A comparison of actigraphy and sleep diaries for infants' sleep behavior. Frontiers In Psychiatry 2015; 6(19).

[24] Vallieres A, Morin C. Actigraphy in the assessment of insomnia. Sleep 2003; 26(7): 902-906. https://doi.org/10.1093/sleep/26.7.902

\section{DOI: https://doi.org/10.6000/2292-2598.2018.06.01.1}

(C) 2018 Kanupka et al.; Licensee Lifescience Global.

This is an open access article licensed under the terms of the Creative Commons Attribution Non-Commercial License (http://creativecommons.org/licenses/by-nc/3.0/) which permits unrestricted, non-commercial use, distribution and reproduction in any medium, provided the work is properly cited. 